\title{
Steroid receptor coactivator AIB1 in endometrial carcinoma, hyperplasia and normal endometrium: correlation with clinicopathologic parameters and biomarkers
}

\author{
Nicole N Balmer ${ }^{1}$, Jennifer K Richer ${ }^{2, *}$, Nicole S Spoelstra ${ }^{2}$, Kathleen C Torkko ${ }^{1}$, \\ Pamela L Lyle ${ }^{3}$ and Meenakshi Singh ${ }^{1}$ \\ ${ }^{1}$ Department of Pathology, University of Colorado Health Sciences Center, Denver, CO, USA; ${ }^{2}$ Department of \\ Medicine, Division of Endocrinology, Metabolism and Diabetes, University of Colorado Health Sciences \\ Center, Denver, CO, USA and ${ }^{3}$ Department of Pathology, Penrose Hospital, Colorado Springs, CO, USA
}

\begin{abstract}
Members of the p160 steroid receptor cofactor family, including AIB1 (Amplified in Breast Cancer 1) (also known as SRC-3/RAC3/ACTR/pCIP/TRAM-1), are of interest in endometrial carcinoma as they affect the function of estrogen (ER) and progesterone receptors (PR). Since it is feasible that alterations in the expression levels of coregulators can either augment ER activity or reduce the ability of PR to oppose ER action in endometrial cancers, our primary aim was to analyze expression of the AIB1 protein in endometrial carcinoma, carcinomaassociated complex atypical hyperplasia, and carcinoma-associated normal endometrium using immunohistochemistry and tissue microarrays. Expression of AIB1 was compared with other biomarkers and clinicopathologic parameters. We also tested AIB1 expression in non-carcinoma associated hyperplastic, normal secretory and proliferative endometrium to determine baseline AIB1 levels. In endometrial carcinoma, there is a higher expression of AIB1 compared to carcinoma-associated complex atypical hyperplasia (0.007) or carcinoma-associated normal endometrium $(<0.001)$. AlB1 expression correlates with older age $(P=0.003)$, peri- or postmenopausal status $(P=0.002)$ and a higher grade of carcinomas $(P=0.04)$. There were no differences in the expression of additional steroid hormone receptor co-activators (SRC-1 and $\mathrm{p300/CBP)}$ and the co-repressor SMRT between histologic categories. AIB1 expression correlated with ER $(r=0.30, P=0.006)$. The strongest correlation was between ER and PR-B isoform nuclear expression $(r=0.52, P<0.0001)$. AlB1 levels were higher in non-carcinoma associated normal and hyperplastic endometrium compared to carcinomaassociated complex atypical hyperplasia and carcinoma-associated normal endometrium, and were the highest in normal secretory endometrium. In conclusion, high AIB1 expression in endometrial carcinoma is associated with parameters of poor prognosis. We propose that when AIB1 is overexpressed in endometrial carcinoma, ER action is augmented, leading to endometrial hyperplasia and progression to malignancy. Future studies correlating expression with response to hormonal therapy may be beneficial.
\end{abstract}

Modern Pathology (2006) 19, 1593-1605. doi:10.1038/modpathol.3800696; published online 15 September 2006

Keywords: AIB1; SRC-3; endometrial carcinoma; complex atypical hyperplasia; steroid receptors; Her-2/neu

Endometrial adenocarcinoma is the most common malignancy of the female genital tract in the United States. It is known to be a hormone-related malignancy, and many of the risk factors for endometrial

Correspondence: Dr M Singh, MD, Department of Pathology, UCHSC at Fitzsimons, Mail Stop 8104, Building RC-1 North Tower, Rm P18-5126, 12800 E. 19th Ave., PO Box 6511, Aurora, CO 80045, USA.

E-mail: meenakshi.singh@uchsc.edu

${ }^{*}$ This author contributed equally to this work.

Received 13 July 2006; revised 8 August 2006; accepted 16 August 2006; published online 15 September 2006 carcinoma are related to the actions of unopposed estrogen. ${ }^{1}$ However, despite the known associations of endometrial carcinoma with estrogen exposure, hormonal therapy is often not as effective in patients with endometrial carcinoma as it is for breast carcinomas, which express estrogen receptors (ERs). ${ }^{2-6}$ The reasons for this are currently not clear. Coactivators and corepressors (collectively termed coregulators) are proteins known to influence the action of both ER and progesterone receptors (PRs) (reviewed in $\mathrm{O}^{\prime} \mathrm{Malley}^{7}$ ). PR is known to oppose ER action in the endometrial epithelium. ${ }^{8,9}$ When the shorter PR isoform, PR-A, is selectively knocked out 
in mice (leaving only the longer PR-B isoform) endometrial hyperplasia results, indicating that it is PR-A that suppresses estrogen-induced uterine epithelial cell proliferation. ${ }^{10,11}$

The p160 steroid receptor cofactor (SRC) family of coactivators can modulate both ER and PR activity; however, it has recently been demonstrated that in the mouse uterus, SRC-1 is the preferred coactivator for PR, ${ }^{12,13}$ while AIB1 (Amplified in Breast Cancer 1) (also known as SRC-3/RAC3/ACTR/pCIP/TRAM1) may exert more of an effect on ER activity. ${ }^{14}$ Thus, it is possible that if AIB1 is overexpressed, ER action could be augmented, leading to endometrial hyperplasia and malignancy. Another possible scenario that could lead to unopposed estrogen action is if levels of the corepressor SMRT, which is necessary for PR-A to oppose ER-mediated proliferation, ${ }^{15,16}$ are lower in endometrial hyperplasia and neoplasia than in normal endometrium.

Since it is feasible that alterations in the expression levels of coregulators can either augment ER activity or reduce the ability of PR to oppose ER action in endometrial cancers, the aim of this study was to analyze steroid hormone coregulator protein expression in a spectrum of neoplastic, preneoplastic and normal endometrium, and to compare this expression to ER $\alpha$, total PR (with an antibody that preferentially targets PRA, but also detects PR-B) and PR-B. AIB1 expression correlates with overexpression of Her-2/neu in breast carcinoma and increased Her-2/neu has been found in type II endometrial carcinomas, ${ }^{17,18}$ therefore we also correlated AIB1 expression with that of Her-2/neu. We correlated AIB1 expression with clinicopathologic parameters of endometrial carcinoma such as tumor stage, grade, and histologic type, and with menopausal status. In addition to AIB1, we examined the steroid hormone receptor co-activators SRC-1 and p300/CBP and the co-repressor SMRT to determine if expression of these proteins is altered in endometrial carcinoma. To accomplish this, we constructed tissue microarrays of carcinoma-associated normal endometrium, carcinoma-associated complex atypical hyperplasia and endometrial carcinoma, and utilized these to conduct an analysis of coregulator protein expression by immunohistochemistry.

It is also known that preneoplastic genetic changes can be present in histologically normal appearing endometrium that is associated spatially and chronologically with endometrial carcinoma and in non-cancer associated simple and complex hyperplasia. ${ }^{19}$ Therefore, we also examined AIB1 expression in non-cancer-associated normal secretory and proliferative endometrium and in non-cancer associated hyperplasias to determine whether there is a variation in AIB1 expression in the cycling endometrium and to establish baseline levels in noncancer-associated endometrium.

\section{Materials and methods}

\section{Patient Characteristics}

All cases of endometrial carcinoma, diagnosed in biopsies, curettings and hysterectomies at the University of Colorado Health Sciences Center between March 1997 and June 2003 were identified in the Department of Pathology database and their reports were reviewed. There were 88 endometrial carcinoma cases with adequate tissue for the construction of the tissue microarrays (Table 1). Data on tumor stage, tumor grade and patient menopausal status at the time of diagnosis were collected. Tumor stage and grade were determined using the AJCC/ FIGO criteria.

\section{Tissue Microarrays}

The tissues used in this study were obtained from formalin-fixed, paraffin-embedded blocks archived in The University of Colorado Health Sciences Center, Department of Pathology, under institutional review board approval. For each case, optimal areas for coring were marked on the $\mathrm{H} \& \mathrm{E}$ slides, in triplicate for carcinoma, and one area each was marked for hyperplasia and normal endometrium, where available. One millimeter diameter cores were obtained from the original paraffin blocks with the MTA1 manual tissue arrayer (Beecher Instruments, Inc., Sun Prairie, WI, USA). The cores were then embedded in paraffin tissue array blocks at predetermined positions. Four micrometer sections were cut from the blocks, and a tape adhesive transfer system was used to transfer the sections onto adhesive coated slides (Instrumedics Inc., Hackensack, NJ, USA). Tissue microarray sections were initially stained with H\&E and reviewed,

Table 1 List of samples in the tissue microarrays $(n=88)$

$E C^{\mathrm{a}}$ only $\quad E C^{\mathrm{a}}$ and $C A$-normal ${ }^{\mathrm{b}} \quad E C^{\mathrm{a}}, C A$-normal ${ }^{\mathrm{b}}, C A-C A H^{\mathrm{c}}$

$E C^{\mathrm{a}}$ and $C A-C A H^{\mathrm{c}}$

CA-normal ${ }^{\mathrm{a}}$ and $C A-C A H^{\mathrm{c}}$

\begin{tabular}{l} 
Number of cases 40 \\
\hline${ }^{\mathrm{a}}$ Endometrial carcinoma. \\
${ }^{\mathrm{b}}$ Carcinoma-associated normal endometrium. \\
${ }^{\mathrm{c}}$ Carcinoma-associated complex atypical hyperplasia.
\end{tabular}


revealing that all 88 cases had adequate tissue for further evaluation.

\section{AIB1 Immunoblot Analysis}

We compared two AIB1 antibodies designed to recognize the AIB1 protein by immunoblot analysis. Both of these have been used in previous studies, in breast tissue. ${ }^{20,21}$ The AIB1 clone 34 (BD Biosciences, San Jose, CA, USA) is a mouse monoclonal IgG antibody generated from a peptide (amino acids 376-389) in the human AIB1 protein. The other AIB1 antibody is NCoA3 C-20 (Santa Cruz Biotechnology, Santa Cruz, CA, USA), a goat polyclonal antibody raised against an undisclosed peptide mapping at the C-terminus of AIB1 of human origin. COS7 cells were transfected with human AIB1 cDNA expression vector in PCR.2.1 (kindly obtained from Bert O’Malley Baylor, Houston, TX, USA) using calcium phosphate precipitation as described previously. ${ }^{16}$ Transfected and untransfected cells were harvested $48 \mathrm{~h}$ after transfection in RIPA buffer. Protein extracts were equalized to $150 \mu \mathrm{g}$ by the Bradford assay (Bio-Rad), resolved by SDS-PAGE and transferred onto nitrocellulose. Equivalent protein loading was confirmed by Ponceau S staining. The Santa Cruz NcoA3 C-20 antibody was utilized as follows: nitrocellulose was blocked with TBS 5\% milk and the primary antibody was used at a 1:250 dilution with TBST (0.05\% Tween) 5\% milk and incubated at $4^{\circ}$ overnight as recommended by the manufacturer. Following three $10 \mathrm{~min}$ washes in TBST, donkey anti-Goat IgG-HRP (Santa Cruz) was used at a 1:15000 dilution in TBST 5\% milk for $45 \mathrm{~min}$ at room temperature. The AIB1 antibody from BD-Biosciences was used under the same conditions except with PBST (0.1\% Tween) and the primary was used at a dilution of 1:250 with $1.5 \%$ milk and goat anti-mouse-HRP antibody (Rockland Immunochemicals, Philadelphia, PA, USA) was used at a 1:1000 dilution in PBST and $3 \%$ milk. Protein bands were detected by enhanced chemiluminescence (Amersham, Arlington Heights, IL, USA).

\section{Immunohistochemistry}

A list of all antibodies used, companies from which they were purchased, and optimized titers are shown in Table 2. For each antibody, optimal titer was determined using full paraffin block sections and then confirmed on tissue microarray sections. Normal and malignant breast and endometrial tissue, and pancreatic carcinoma sections were used as controls. All tissue microarray sections were stained using the Autostainer Universal Staining System from DakoCytomation (Carpinteria, CA, USA).

\section{AIB1 Santa Cruz Biotechnology Antibody (SC)}

Slides were deparaffinized using xylene washes, and were rehydrated through graded alcohols. Antigen retrieval was carried out using a $10 \mathrm{mM}$ sodium citrate (pH 6.0) solution and microwave heating to a temperature of $95^{\circ} \mathrm{C}$ for $10 \mathrm{~min}$. Blocking of endogenous peroxidase activity was performed with $0.3 \% \mathrm{H}_{2} \mathrm{O}_{2}$, and nonspecific reactions were blocked with $1.5 \%$ donkey serum in PBS for $1 \mathrm{~h}$ (Santa Cruz Biotechnology). Primary antibody was applied and sections were incubated at room temperature for $1 \mathrm{~h}$. For negative control sections, primary antibody was replaced with non-immune goat serum. Antibody was detected using the Goat ABC staining system (Santa Cruz Biotechnology). Slides were counterstained with Gills hematoxylin, dehydrated and coverslipped.

\section{AIB1 BD Biosciences Antibody (BD), and Antibodies for CBP, SRC-1 and SMRT}

Slides were deparaffinized using xylene washes, and rehydrated through graded alcohols. Antigen retrieval was carried out using a Biocare Medical Decloaker (Concord, CA, USA) using $1 \times$ Target Retrieval Solution (DakoCytomation) for $5 \mathrm{~min}$ at $120^{\circ} \mathrm{C}$. Blocking of endogenous peroxidase activity was performed with $3 \% \mathrm{H}_{2} \mathrm{O}_{2}$ for 5-10 min. Primary antibody was applied and sections were incubated

Table 2 Immunohistochemistry antibody data

\begin{tabular}{lll}
\hline & Clone & Source \\
\hline AIB1 (SC $)$ & Goat (C20) & Santa Cruz Biotechnology, Santa Cruz, CA, USA \\
AIB1 (BD $)$ & 34 & BD Biosciences, San Jose, CA, USA \\
Her2-neu & Rabbit & DakoCytomation, Carpinteria, CA, USA \\
E3- (alpha) & DD5 & DakoCytomation, Carpinteria, CA, USA \\
PR (total) & PgR 636 & DakoCytomation, Carpinteria, CA, USA \\
PR-B & hPRa6 & LabVision, Fremont, CA, USA \\
CBP & Rabbit & Upstate, Lake Placid, NY, USA \\
SRC-1 & $1135 / \mathrm{H} 4$ & Affinity BioReagents, Golden, CO, USA \\
SMRT & 44 & BD Biosciences, San Jose, CA, USA \\
\end{tabular}

${ }^{\mathrm{a}}$ Santa Cruz.

$\mathrm{b}_{\mathrm{BD}}$ Bioscience. 
at room temperature for $1 \mathrm{~h}$. For negative control sections, primary antibody was replaced with non-immune mouse serum (BD Biosciences). The Vectastain Elite ABC Universal kit (Vector Labs, Burlingame, CA, USA) was applied at room temperature using $30 \mathrm{~min}$ for each reagent. DAB + (DakoCytomation) was applied for 4 min. Slides were counterstained with hematoxylin, dehydrated and coverslipped.

\section{ER-Alpha, PR (Total PR and Isoform PR-B) and Her-2/neu}

For ER, total PR, PR-B and Her-2/neu, slides were treated as above with the exception that antigen retrieval for Her-2/neu was performed at $105^{\circ} \mathrm{C}$ for $20 \mathrm{~min}$, and the detection system for all the slides was Envision (DakoCytomation), which was applied for $30 \mathrm{~min}$.

\section{Scoring and Analysis of Immunohistochemistry Sections}

The arrays were graded on a 0 to $3+$ intensity scale for all the biomarkers. The percentage of cells staining with a given intensity was recorded for each antibody. A minimum of $10 \%$ of the tumor cells staining with the given intensity was necessary to be included within that intensity cohort. Staining scores (range 0-300) were calculated for each marker by multiplying the intensity score (0-3) by the percent of cells staining (0-100). Separate scores were given for carcinoma, and for hyperplastic and normal endometrium for each case, when present. Her-2/neu stained sections were scored with regard to cell membrane staining, on a 0 to $3+$ scale based on FDA approved guidelines for interpretation of HercepTest ${ }^{\mathrm{TM}}$ stained slides; whereby 0 and $1+$ scores were considered as negative results and $2+$ and $3+$ as positive. Scoring was performed simultaneously by two pathologists (MS and NB) on a multihead scope. Discordant scores were resolved by consensus.

\section{Statistical Analyses}

The paired signed rank test was used to test score differences between cancer, normal and hyperplastic endometrium in the same patient. Either the Wilcoxon rank sum or Kruskal-Wallis tests were used to test for differences in scores and the relationship of expression of these markers to clinical and pathologic parameters. Significance was determined by $P$-values less than 0.05 and all statistical analyses were performed using SAS software version 9.1 (SAS Institute, Cary, NC, USA). Statistics were performed for all stains, and all categories. A Bonferroni correction was used to adjust $P$-values for multiple comparisons. Where $P$-values are between 0.02 and 0.05 , the significance should be interpreted conservatively. Correlations between expression scores for different proteins were tested using Spearman's correlation coefficient.

\section{Results}

\section{Clinicopathologic Characteristics}

Of the 88 patients studied by tissue microarray, $71(81 \%)$ were age 50 years or older, with $63(72 \%)$ being postmenopausal, 10 (11\%) perimenopausal and $15(17 \%)$ premenopausal. The average age of the patients in our study was 60.0 years, with a range of 33 years to 88 years. Forty of the 88 cases $(45 \%)$ had endometrial carcinoma only, whereas the other 48 (55\%) had carcinoma-associated normal and/or carcinoma-associated complex atypical hyperplasia in the tissue microarray (Table 1). In addition, full sections from five cases each of non-cancer associated normal secretory endometrium, normal proliferative endometrium, simple hyperplasia and complex hyperplasia $(n=20)$ were stained with AIB1 to study a full spectrum of benign and malignant endometrium.

Fifty-nine $(67 \%)$ of the cases were stage I, with six $(7 \%)$ being stage II, nine $(10 \%)$ stage III and three $(3 \%)$ stage IV. Staging information was not available for $11(13 \%)$ of the cases as these patients had further clinical care performed elsewhere. Forty-eight of the cases (55\%) were grade I, 23 $(26 \%)$ were grade II and 17 of the cases (19\%) were grade III. Seventy $(80 \%)$ of the carcinomas had endometriod histology, three (3\%) were mucinous adenocarcinoma, three (3\%) were undifferentiated carcinomas, one (1\%) was pure papillary serous carcinoma, and 11 (13\%) were endometriod carcinomas with a component of papillary serous or clear cell carcinoma. There were no pure clear cell carcinoma cases. Tumors were categorized as Type I or Type II based on histologic type. All endometrioid (Grade I-III) and mucinous carcinomas were grouped as Type I carcinoma. Pure papillary serous carcinoma, carcinomas with a component of papillary serous and clear cell components, and undifferentiated carcinomas were grouped as Type II carcinomas.

It is known that complex atypical hyperplasia is most often associated with Type I endometrial tumors. This was true for this study also, with 23 $(82 \%)$ of the complex atypical hyperplasias in the tissue microarrays being from patients with Type I carcinoma. The presence of carcinoma-associated complex atypical hyperplasia from patients in this study with Type II carcinoma is explained by the fact that 11 of our 15 Type II cases (73\%) had an endometriod component also in addition to clear cell or papillary serous histology. Therefore, it is likely that the carcinoma-associated complex atypical hyperplasia is associated with the endometrioid component of the tumor in these cases. 


\section{AIB1 Immunoblot Analysis}

In an attempt to understand the differences, we saw in the AIB1 protein expression patterns on immunohistochemistry with the two AIB1 antibodies, Western blot analysis was performed on protein extract from cells transfected with expression vector encoding human AIB1 vs untransfected cells. Results from the immunoblot (Figure 1) demonstrated that the AIB1 (BD) antibody detected a single protein band migrating according to the appropriate molecular weight $(160 \mathrm{kDa})$ of the AIB1 protein only in cells transfected with AIB1 and not in the untransfected cells. In addition to a band migrating at the appropriate molecular weight, the Santa Cruz antibody more strongly detected an additional protein. This result may suggest that the cytoplasmic staining observed when this antibody is used for immunohistochemistry could result from crossreactivity to a nonspecific protein. Therefore, only the results of the stains using the AIB1 (BD) antibody are discussed in detail; however, the AIB1 (SC) results are also summarized briefly.

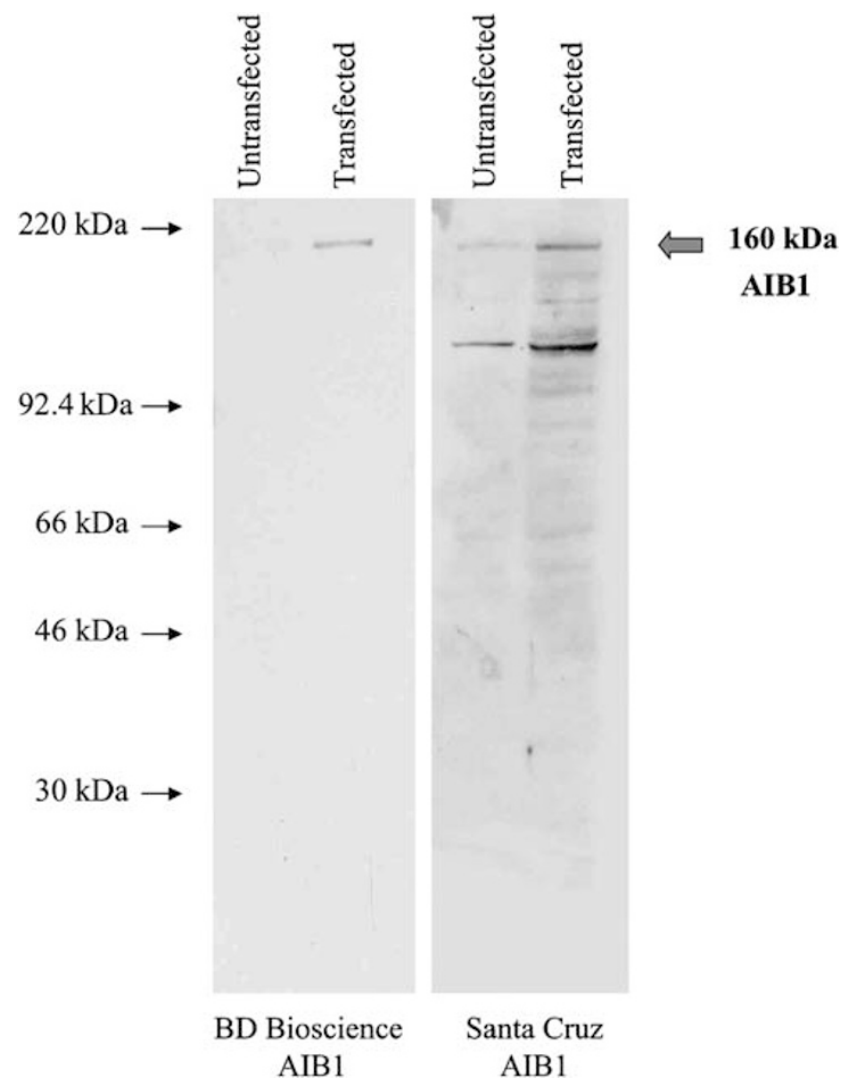

Figure 1 Immunoblot of AIB1 (BD) and AIB (SC) antibodies. The AIB1 (BD) antibody detected a single protein band migrating according to the appropriate molecular weight $(160 \mathrm{kDa})$ of the AIB1 protein only in cells transfected with AIB1/SRC-3 and not in the untransfected cells. In addition to a band migrating at the appropriate molecular weight, the AIB1 (SC) antibody more strongly detected an additional protein.

\section{Expression Patterns}

Nuclear expression was noted for ER (Figure 2a-c) and PR total (Figure 2d-f). Both nuclear and cytoplasmic staining is expected in $\mathrm{PR}-\mathrm{B},{ }^{22}$ and since the two cellular locations could potentially have different correlations with AIB1 expression and prognostic factors, nuclear and cytoplasmic staining were both scored separately (Figure $2 \mathrm{~g}-\mathrm{i}$ ). AIB1 showed a nuclear pattern of expression using the AIB1 (BD) antibody (Figure 3a-d) and a cytoplasmic pattern of expression using the AIB1 (SC) antibody (data not shown). Her-2/neu protein had a cell membrane pattern of overexpression (Figure 2j-l). The expression of CBP, SRC-1 and SMRT was predominantly nuclear.

\section{AIB1 Expression in Non-Cancer Associated Normal Endometrium}

AIB1 was expressed in $100 \%$ of the cases of noncancer-associated normal endometrium and hyperplasia. The mean expression scores were higher in them than in normal and hyperplastic tissue from patients with endometrial carcinoma and are as follows: normal secretory $=249$, normal proliferative $=202$, simple hyperplasia $=192$ and complex hyperplasia $=187$.

AIB1 Expression in Endometrial Carcinoma vs Carcinoma-Associated Complex Atypical Hyperplasia vs Carcinoma-Associated Normal Endometrium (Table 3)

Type I and Type II endometrial carcinoma combined AIB1 expression using the AIB1 (BD) antibody was the highest in endometrial carcinoma, lesser in carcinoma-associated complex atypical hyperplasia, and the least in carcinoma-associated normal endometrium (Table 3). (Mean scores for endometrial carcinoma, carcinoma-associated complex atypical hyperplasia and carcinoma-associated normal endometrium, respectively, are equal to 201.6, 141, 121.) AIB1 was expressed in 79 of 82 endometrial carcinoma cases $(93 \%), 24$ of $28(86 \%)$ carcinomaassociated complex atypical hyperplasia and 36 of $41(88 \%)$ of carcinoma-associated normal endometrium. There was a significantly higher expression, using median values of the expression scores of AIB1 in endometrial carcinoma compared to carcinoma-associated complex atypical hyperplasia $(P=0.007)$ and endometrial carcinoma compared to carcinoma-associated normal endometrium $(P<0.001)$. There was no significant difference in the expression of AIB1 between carcinoma-associated complex atypical hyperplasia vs carcinomaassociated normal endometrium.

\section{Type 1 endometrial carcinoma}

Different expression profiles were present for AIB1 when comparing Type I endometrial carcinoma 

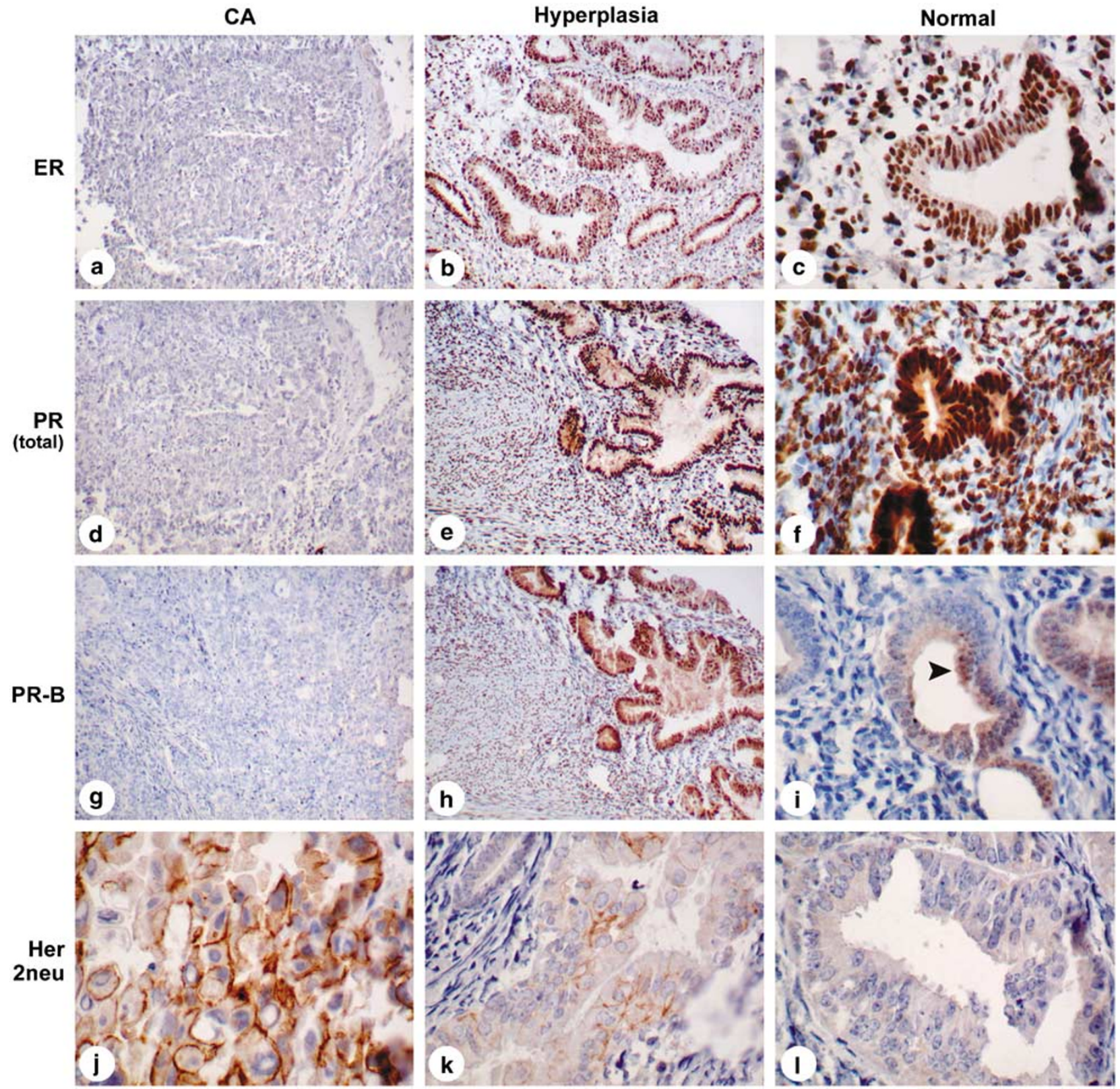

Figure 2 ER, PR(total), PR-B and Her-2/neu. (a-c) ER (alpha): nuclear expression. (a) No ER expression in endometrial carcinoma (negative) $(\times 40)$. (b) ER expression in carcinoma-associated complex atypical hyperplasia, $2+$ positive $(\times 40)$. $(\mathbf{c})$ ER expression in carcinoma-associated normal endometrium, $3+$ positive $(\times 400)$. $(\mathbf{d}-\mathbf{f})$ PR (total): nuclear expression. (d) No PR expression in endometrial carcinoma (negative) $(\times 40)$. (e) PR expression in carcinoma-associated complex atypical hyperplasia, $3+$ positive $(\times 40)$. (f) PR expression in carcinoma-associated normal endometrium, $3+$ positive $(\times 400)$. (g-i) PR-B: nuclear and cytoplasmic expression. (g) No PR-B expression in endometrial carcinoma (negative) $(\times 40)$. (h) PR-B expression in carcinoma-associated complex atypical hyperplasia, $3+$ positive, predominantly nuclear expression $(\times 40)$. (i) PR-B expression in carcinoma-associated normal endometrium, $2+$ positive, cytoplasmic expression (arrow head) $(\times 400)$. (j-k) Her 2/neu protein overexpression: cell membrane pattern. (j) Her $2 /$ neu overexpression in endometrial carcinoma, $3+(\times 400)$. $(\mathbf{k})$ Her $2 /$ neu overexpression in carcinoma-associated complex atypical hyperplasia, $2+(\times 200)$. (l) No Her $2 /$ neu overexpression in carcinoma-associated normal endometrium, $(0)(\times 200)$. CA $=$ endometrial carcinoma.

$(n=72)$ with carcinoma-associated normal endometrium and carcinoma-associated complex atypical hyperplasia. AIB1 expression was significantly higher/different in Type I endometrial carcinoma $(n=72)$ compared to carcinomaassociated normal endometrium $(P=0.0002)$, and in carcinoma-associated complex atypical hyperplasia compared to carcinoma-associated normal endometrium $(P=0.016)$, but not in Type I endometrial carcinoma compared to carcinomaassociated complex atypical hyperplasia (Table 3). This is consistent with the difficulty that a 

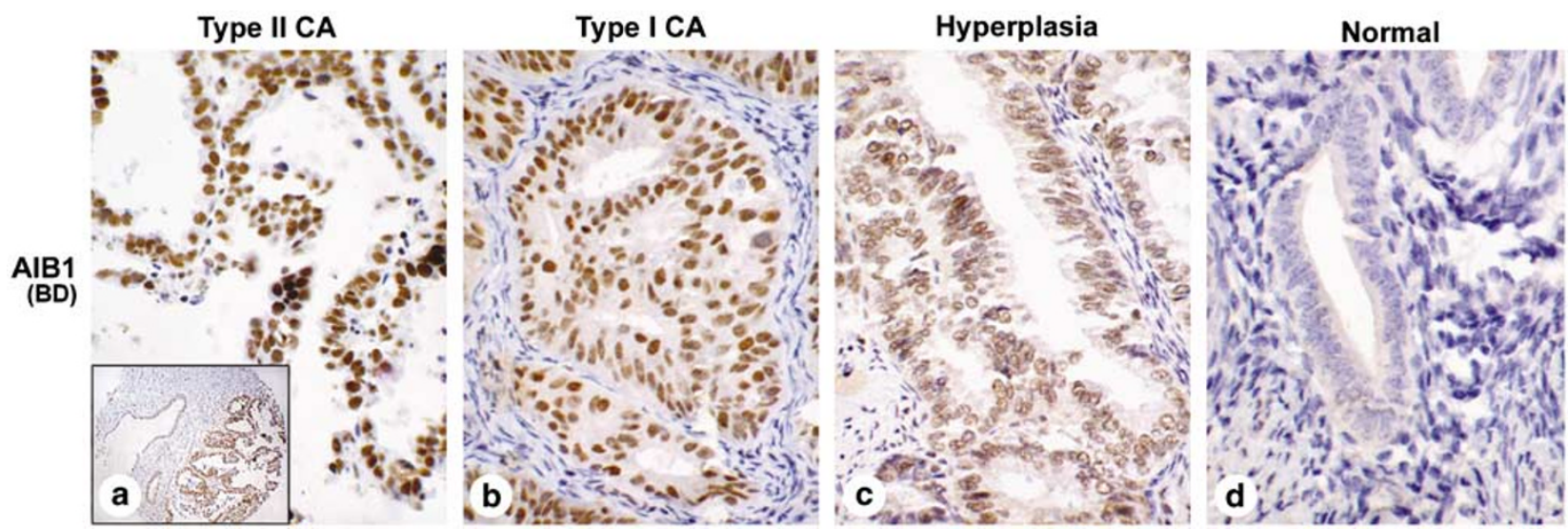

Figure 3 AIB1. (a-d) AIB1 (BD): nuclear expression. (a) AIB1 (BD) expression in Type II endometrial carcinoma, $3+$ positive ( $\times 200$ ). (b) AIB1 (BD) expression in Type I endometrial carcinoma, $2+$ positive $(\times 200)$. (c) AIB1 (BD) expression in carcinoma-associated complex atypical hyperplasia, $1+$ positive $(\times 200)$. (d) AIB1 (BD) No expression in carcinoma-associated normal endometrium (negative) $(\times 200)$. $\mathrm{CA}=$ endometrial carcinoma.

Table 3 Expression profiles-endometrial carcinoma vs carcinoma-associated complex atypical hyperplasia vs carcinoma-associated normal endometrium

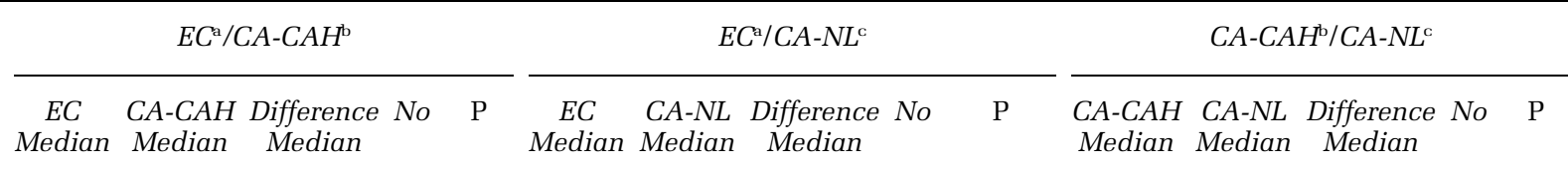

All carcinoma types combined

$\begin{array}{lcrrrrr}\text { AIB1 }\left(\mathrm{BD}^{\mathrm{d}}\right) & 200 & 180 & 62.5 & 27 & \mathbf{0 . 0 0 7} & 200 \\ \text { Her2neu } & 1 & 1 & 0 & 25 & \mathbf{0 . 0 3 6} & 1 \\ \text { PRB-N } & 135 & 200 & -55 & 24 & \mathbf{0 . 0 4 1} & 90 \\ \text { PRB-C } & 180 & 200 & 0 & 24 & 0.775 & 185 \\ \text { ER } & 260 & 300 & -30 & 25 & 0.064 & 250 \\ \text { PR (total) } & 257.5 & 300 & 0 & 24 & 0.498 & 240\end{array}$

$\begin{array}{rrrcr}90 & 60 & 37 & <\mathbf{0 . 0 0 0 1} & 190 \\ 0 & 1 & 30 & \mathbf{0 . 0 0 0 2} & 0 \\ 300 & -120 & 33 & <\mathbf{0 . 0 0 0 1} & 100 \\ 200 & -50 & 34 & \mathbf{0 . 0 1 4} & 250 \\ 300 & -30 & 31 & <\mathbf{0 . 0 0 0 1} & 300 \\ 300 & -42.5 & 30 & \mathbf{0 . 0 0 3} & 285\end{array}$

$\begin{array}{rrrr}150 & 10 & 18 & 0.072 \\ 0 & 0 & 11 & 0.500 \\ 300 & 0 & 9 & 0.125 \\ 300 & 0 & 10 & 0.563 \\ 300 & 0 & 9 & 0.500 \\ 300 & -10 & 10 & 0.313\end{array}$

Type I carcinomas

\begin{tabular}{|c|c|c|c|c|c|}
\hline AIB1 $\left(B^{d}\right)$ & 200 & 160 & 20 & 20 & 0.107 \\
\hline Her2neu & 1 & 1 & 0 & 20 & 0.016 \\
\hline PRB-N & 120 & 200 & -70 & 20 & 0.056 \\
\hline PRB-C & 145 & 200 & 0 & 20 & 0.519 \\
\hline ER & 225 & 300 & -30 & 20 & 0.040 \\
\hline PR (total) & 250 & 300 & 0 & 20 & 0.636 \\
\hline \multicolumn{6}{|c|}{ Tyре II carcinomas } \\
\hline $\mathrm{AIB} 1\left(\mathrm{BD}^{\mathrm{d}}\right)$ & 300 & 180 & 120 & 7 & 0.063 \\
\hline Her2neu & 0 & 0 & 0 & 5 & 1.00 \\
\hline PRB-N & 195 & 230 & 10 & 4 & 1.00 \\
\hline PRB-C & 272.5 & 245 & 47.5 & 4 & 0.500 \\
\hline ER & 270 & 300 & 0 & 5 & 0.750 \\
\hline PR (total & 270 & 250 & -7.5 & 4 & 0.75 \\
\hline \multicolumn{6}{|c|}{$\begin{array}{l}\mathrm{PRB}-\mathrm{N}=\mathrm{PR}-\mathrm{B} \text { isoform nuclear expression; } \mathrm{PRB}-\mathrm{C}=\mathrm{PR} \\
\text { both types of tissue. }\end{array}$} \\
\hline \multicolumn{6}{|c|}{${ }^{\mathrm{a}}$ Endometrial carcinoma; } \\
\hline \multicolumn{6}{|c|}{ bCarcinoma-associated complex atypical hyperplasia; } \\
\hline \multicolumn{6}{|c|}{$\begin{array}{l}{ }^{\mathrm{c}} \text { Carcinoma-associated normal endometrium. } \\
\mathrm{d}_{\mathrm{BD}} \text { Bioscience. }\end{array}$} \\
\hline
\end{tabular}

Bold values indicate statistically significant $P$-values.

pathologist encounters in differentiating complex atypical hyperplasia from Type I endometrial carcinoma when using cytological features alone, thus necessitating reliance on architectural features to assist in differentiation between these two entities. 
Table 4 Biomarker expression: correlation with clinicopathologic parameters in endometrial carcinoma

\begin{tabular}{|c|c|c|c|c|c|c|c|c|c|c|c|c|c|c|c|c|c|c|c|c|}
\hline & \multicolumn{4}{|c|}{$A I B 1\left(B D^{\mathrm{a}}\right)$} & \multicolumn{4}{|c|}{$E R$} & \multicolumn{4}{|c|}{$P R$ (total) } & \multicolumn{4}{|c|}{ PRB-N } & \multicolumn{4}{|c|}{ PRB-C } \\
\hline & $\mathrm{n}$ & Median & $\begin{array}{c}K-W \\
\mathrm{P}\end{array}$ & $\begin{array}{c}\text { WRS } \\
\mathrm{P}\end{array}$ & $\mathrm{n}$ & Median & $\begin{array}{c}K-W \\
\mathrm{P}\end{array}$ & $\begin{array}{c}\text { WRS } \\
\mathrm{P}\end{array}$ & $\mathrm{n}$ & Median & $\begin{array}{c}K-W \\
\mathrm{P}\end{array}$ & $\begin{array}{c}\text { WRS } \\
\mathrm{P}\end{array}$ & $\mathrm{n}$ & Median & $\begin{array}{c}K-W \\
\mathrm{P}\end{array}$ & $\begin{array}{c}\text { WRS } \\
\mathrm{P}\end{array}$ & $\mathrm{n}$ & Median & $\begin{array}{c}K-W \\
\mathrm{P}\end{array}$ & $\begin{array}{c}W R S \\
\mathrm{P}\end{array}$ \\
\hline \multicolumn{21}{|c|}{ Menopause } \\
\hline Pre & 13 & 80 & 0.002 & & 14 & 265 & 0.84 & & 15 & 255 & 0.28 & & 14 & 105 & 0.79 & & 14 & 200 & 0.42 & \\
\hline Peri & 10 & 200 & & & 10 & 210 & & & 10 & 255 & & & 10 & 100 & & & 10 & 170 & & \\
\hline Post & 59 & 200 & & & 60 & 210 & & & 60 & 202.5 & & & 61 & 120 & & & 61 & 170 & & \\
\hline \multicolumn{21}{|l|}{ Stage } \\
\hline I & 54 & 200 & & 0.09 & 55 & 230 & & 0.02 & 56 & 240 & & 0.004 & 56 & 130 & & 0.31 & 56 & 195 & & 0.69 \\
\hline II-IV & 18 & 190 & & & 18 & 160 & & & 18 & 120 & & & 18 & 85 & & & 18 & 160 & & \\
\hline \multicolumn{21}{|l|}{ Grade } \\
\hline & 43 & 200 & & 0.04 & 45 & 250 & & 0.01 & 46 & 247.5 & & 0.03 & 45 & 120 & & 0.42 & 45 & 150 & & 0.05 \\
\hline II/III & 38 & 270 & & & 38 & 200 & & & 38 & 195 & & & 39 & 100 & & & 39 & 200 & & \\
\hline \multicolumn{21}{|c|}{ Histo type } \\
\hline I & 67 & 200 & & 0.07 & 68 & 210 & & 0.48 & 69 & 210 & & 0.21 & 69 & 120 & & 0.09 & 69 & 170 & & 0.08 \\
\hline II & 14 & 270 & & & 15 & 270 & & & 15 & 120 & & & 15 & 180 & & & 15 & 200 & & \\
\hline
\end{tabular}

$\mathrm{K}-\mathrm{W}=$ Kruskal-Wallis test; WRS $=$ Wilcoxon rank sum test; PRB-N = PR-B isoform nuclear expression; PRB-C = PR-B cytoplasmic expression. ${ }^{\mathrm{a}}$ BD Bioscience.

Bold values indicate statistically significant $P$-values.

Type II endometrial carcinoma

The difference in AIB1 expression when comparing Type II endometrial carcinoma $(n=15)$ to carcinoma-associated complex atypical hyperplasia and carcinoma-associated normal endometrium approached but did not reach significance $(P=0.063$ for both; Table 3).

\section{AIB1 Correlation with Known Histologic and Clinical Parameters (Table 4)}

There was a trend for lower AIB1 expression in Type I vs Type II endometrial carcinoma $(P=0.07)$. AIB1 expression correlated significantly with perimenopausal and postmenopausal status $(P=0.002)$ and showed a significantly higher expression in higher grade (grade II and III) compared to lower grade (I) carcinomas $(P=0.04)$. AIB1 expression did not correlate with surgical stage.

\section{Summary of AIB1 (SC) Immunohistochemistry Results}

AIB1 as detected by the AIB1 (SC) antibody was expressed in 82 of $84(97 \%)$ endometrial carcinoma cases, 21 of 26 (81\%) carcinoma-associated complex atypical hyperplasia samples and in 18 of $38(47 \%)$ carcinoma-associated normal endometrium samples in the tissue microarrays. The mean scores were as follows: endometrial carcinoma $=173.3$, carcinomaassociated complex atypical hyperplasia $=86$ and carcinoma-associated normal endometrium $=44$. AIB1 detected with the AIB1(SC) antibody exhibited significant differences in expression for endometrial carcinoma compared to carcinoma-associated complex atypical hyperplasia $(P=0.0008)$ and endometrial carcinoma compared to carcinomaassociated normal endometrium $(P<0.0001)$. In addition, AIB1(SC) expression was significantly lower in Type I than Type II cancers. In contrast to AIB1 detected with the AIB1(BD) antibody, AIB1 expression as detected with the AIB1(SC) antibody did not correlate with higher grade tumors. AIB1 expression using either antibody did not correlate with higher surgical stage.

\section{Additional Coactivators of Steroid Hormone Receptors (SRC-1 and p300/CBP) and Co-repressor (SMRT) Studied by Immunohistochemistry on Tissue Microarrays}

None of the other co-activators (CBP and SRC-1) or the co-repressor (SMRT) evaluated by immunohistochemistry showed differences in expression in carcinoma-associated normal endometrium, carcinoma-associated complex atypical hyperplasia or endometrial carcinoma tissues or had any correlation with tumor grade and histologic type (data not shown).

\section{ER and PR (Total) Expression in Endometrial Carcinoma vs Carcinoma-Associated Complex Atypical Hyperplasia vs Carcinoma-Associated Normal Endometrium (Table 3)}

Type I and II endometrial carcinoma combined ER and PR (total) exhibited significantly higher expression in carcinoma-associated normal endometrium compared with Type I and Type II 
endometrial carcinoma combined $(P<0.0001$ and 0.003 , respectively). There was a trend toward a higher expression of ER in carcinoma-associated complex atypical hyperplasia (median $=300$ ) compared to Type I and II endometrial carcinoma combined (median $=260$ ), but this was not statistically significant. There was no difference in ER or PR (total) expression in carcinoma-associated complex atypical hyperplasia tissue compared with carcinoma-associated normal endometrium.

\section{Type I endometrial carcinoma}

Similarly, ER and PR (total) both exhibited higher expression in carcinoma-associated normal endometrium compared to Type I endometrial carcinoma $(P=0.0001$ and 0.012 , respectively). In addition, ER was expressed significantly more in carcinomaassociated complex atypical hyperplasia than in Type I endometrial carcinoma $(P=0.040)$.

\section{Type II endometrial carcinoma}

The differences in ER and total PR expression for carcinoma-associated normal endometrium and carcinoma-associated complex atypical hyperplasia compared to Type II endometrial carcinoma were not significant, though a trend towards higher steroid receptor expression in benign tissue persisted (Table 3).

\section{ER and Total PR Correlation with Known Clinicopathologic Parameters (Table 4)}

ER and PR (total) expression was significantly greater in stage I tumors as compared with higher stage (II-IV) tumors $(P=0.02$ and 0.004 , respectively). In addition, both ER and PR (total) exhibited higher expression in grade I vs higher grade (II and III) tumors $(P=0.01$ and 0.03$)$.

PR-B Expression in Endometrial Carcinoma vs Carcinoma-Associated Complex Atypical Hyperplasia vs Carcinoma-Associated Normal Endometrium (Table 3)

\section{Type I and II endometrial carcinoma}

PR-B staining was evaluated separately for nuclear and cytoplasmic expression. There was a significantly higher expression in carcinoma-associated normal endometrium compared to all Type I and II endometrial carcinoma combined, with both staining patterns (nuclear: $P<0.0001$; cytoplasmic: $P=0.014)$. PR-B expression was also higher in carcinoma-associated complex atypical hyperplasia compared to endometrial carcinoma, but this relationship was significant only for nuclear staining $(P=0.041)$. There was no difference in expression between carcinoma-associated complex atypical hyperplasia compared to carcinoma-associated normal endometrium for PR-B nuclear or cytoplasmic staining.
Type I endometrial carcinoma

PR-B expression in benign tissue compared to Type I carcinoma was similar to that of Type I and II endometrial carcinoma combined, with significantly higher expression in carcinoma-associated normal endometrium compared to Type I carcinoma for both staining patterns (nuclear: $P<0.0001$; cytoplasmic: $P=0.036$ ). In addition, there was a trend toward higher PR-B expression in carcinoma-associated complex atypical hyperplasia compared to Type I endometrial carcinoma, but this difference was not significant for either staining pattern.

Type II endometrial carcinoma

PR-B expression was not significantly different between carcinoma-associated normal endometrium and carcinoma-associated complex atypical hyperplasia compared to Type II endometrial carcinoma for either staining pattern (Table 3).

\section{PR-B Correlation with Known Clinicopathologic Parameters (Table 4)}

Neither of the PR-B staining patterns correlated significantly with patient menopausal status, tumor stage, tumor grade or tumor histologic type. However, both PR-B nuclear and PR-B cytoplasmic staining showed a trend towards lower expression in Type I endometrial carcinoma compared to Type II endometrial carcinoma $(P=0.09$ and 0.08 , for nuclear and cytoplasmic staining, respectively).

\section{Her-2/neu Expression in Endometrial Carcinoma vs Carcinoma-Associated Complex Atypical Hyperplasia vs Carcinoma-Associated Normal Endometrium (Table 3)}

Her-2/neu exhibited significantly higher expression in Type I and II endometrial carcinoma combined compared with carcinoma-associated normal endometrium $(P=0.0002)$, and in Type I and II endometrial carcinoma combined compared with carcinoma-associated complex atypical hyperplasia $(P=0.036)$. There was no difference in Her-2/ neu expression in carcinoma-associated complex atypical hyperplasia compared with carcinomaassociated normal endometrium. Her-2/neu also exhibited higher expression in Type I endometrial carcinoma compared to carcinoma-associated normal endometrium $(P=0.0003)$ and carcinomaassociated complex atypical hyperplasia $(P=0.016)$. There was no difference in Her-2/neu expression in Type II endometrial carcinoma compared to carcinoma-associated normal endometrium or carcinoma-associated complex atypical hyperplasia (Table 3). 
Her-2/neu Correlation with Known Clinicopathologic Parameters

Her-2/neu expression did not correlate with patient menopausal status, stage, or the histologic type and grade of tumor.

\section{Comparison of all Biomarkers}

There were significant differences between the relative expression of all the biomarkers in carcinoma-associated normal endometrium, carcinomaassociated complex atypical hyperplasia and Type I and II endometrial carcinoma combined ( $P$-values for all <0.001) with the only exception being the cytoplasmic expression on PR-B which was not as significant $(P=0.04)$.

\section{Correlations between Biomarkers}

The strongest correlation was between ER and the PR-B-nuclear (Spearman correlation coefficient, $r=0.52, P<0.0001)$. AIB1 expression as determined by the BD antibody correlated with ER $(r=0.30$, $P=0.006$ ). There was no evidence of correlation between AIB1 expression using the SC antibody and the other biomarkers, including AIB1 expressed using the BD antibody, further indicating that this particular antibody did not specifically detect AIB1.

\section{Discussion}

\section{The Normal Endometrium}

In the normal endometrium, AIB1 protein levels have been found to increase during the late secretory phase, but none of the other coactivators have been reported to be hormonally regulated. ${ }^{23}$ Our results in the noncarcinoma-associated cases correlate with these findings, as the highest AIB1 expression was in normal secretory endometrium, when both estrogen and progesterone levels are higher than they are in the proliferative phase. Vienon et al found that AIB1 mRNA was expressed in normal endometrium and, in contrast to our findings, reported that it did not appear to be hormonally regulated. However, they did note individual variations in expression levels, which they hypothesized to be responsible for differences in response to hormone-based therapies in endometrial carcinomas. ${ }^{24}$ Our study found that AIB1 expression is higher on average in the non-carcinoma associated tissues than in the carcinoma-associated complex atypical hyperplasia and carcinoma-associated normal endometrium. The biologic reasons for this are not entirely clear, but possible explanations include a loss of hormonal regulation in the carcinoma-associated cases and this concept may be further explored in future studies.

\section{Co-Activator and Co-Repressor Expression in Endometrial Carcinomas}

This study reveals increased expression of AIB1 protein in endometrial carcinoma compared to carcinoma-associated complex atypical hyperplasia and carcinoma-associated normal endometrium. Ours is the only study to examine AIB1 at the protein level in endometrial cancers, hyperplasia and the normal endometrium. SRC-1, 2 and 3 proteins were previously examined by immunohistochemistry in the normal endometrium and the endometrium of women with polycystic ovarian syndrome, a group that has a higher likelihood of developing estrogen-induced endometrial hyperplasia and cancer, where AIB1 and SRC-2 were found to be elevated. ${ }^{23}$ An additional study examined SRC-1, p300/CBP, and the co-repressors N-CoR and SMRT, but not AIB1, and found that both SRC-1 and p300/ CBP were reduced in endometrial carcinoma as compared with normal endometrium and hyperplastic endometrium. ${ }^{25}$ In contrast, we observe no change in SRC-1 and CBP/p300 in endometrial carcinoma or carcinoma-associated complex atypical hyperplasia vs the carcinoma-associated normal endometrium. At the mRNA transcript level, SRC-1, 2 and 3 and the co-repressors N-CoR and SMRT were measured in endometrial carcinoma and all were upregulated in malignant endometrium. ${ }^{26}$ However, at the protein level, we find that AIB1 is overexpressed in endometrial cancers relative to hyperplastic or normal endometrium, but SRC-1 and SMRT are not. Glaesar et $a l^{27}$ found AIB1 to be overexpressed in $17 \%$ of endometrial cancers, using PCR, RT-PCR and fluorescent DNA technology. The fact that we see overexpression at the protein level in a higher percentage of endometrial cancers indicates that an additional mechanism besides gene amplification may be causing overexpression of AIB1.

\section{Correlation with Clinical Parameters}

In addition to demonstrating AIB1 overexpression in endometrial carcinoma and carcinoma-associated complex atypical hyperplasia, we found a correlation between AIB1 expression and clinicopathologic parameters of unfavorable prognosis. Factors such as histologic type, grade and surgical stage of the carcinomas are known to affect prognosis for patients with endometrial carcinoma. We found that high expression of AIB1 correlated with peri- and postmenopausal status and with a higher grade of carcinoma. We did not find correlation with surgical stage. AIB1 demonstrated higher expression in Type II than Type I endometrial carcinoma. This finding is not surprising given the relationship between AIB1 expression and tumor grade. Type II endometrial carcinomas are considered high-grade carcinomas, and are known to have a worse prognosis, indicating that AIB1 is expressed more in aggressive endometrial tumors. It is unclear why AIB1 did not 
correlate with surgical stage in the present study and others, since this parameter is frequently used along with grade to assess prognosis. However, since $83 \%$ of the cases in our study were stage I or II, it is possible that many tumors in our population, including those with a higher grade, were detected before extensive spread had occurred.

\section{Specificity of AIB1 (BD) vs AIB1 (SC) Antibodies}

Our immunoblot analysis tested the ability of these two antibodies to recognize AIB1 specifically, and indicates that the AIB1(BD) antibody, which showed nuclear expression by immunohistochemistry, has more specificity than the AIB1(SC) antibody, which exhibited cytoplasmic expression. We report this result because the AIB1(SC) antibody has been utilized for immunohistochemistry in previous published studies, albeit in the breast. ${ }^{20,21}$ Based upon the known biology of the SRC family of coactivators, it makes sense that the AIB1(BD) nuclear expression is more relevant. The p160 family members are known to work by interacting with ligand-bound nuclear receptors to recruit methyl-transferases and histone acetyl-transferases to specific promoter regions. This results in chromatin remodeling, assembly of transcription factors and transcription of target genes. ${ }^{28,29}$ With this mechanism of action, one would expect to see AIB1 in the nucleus. In addition, other studies examining AIB1 in the endometrium have demonstrated nuclear expression. ${ }^{23}$

\section{Her-2/neu}

Overexpression of Her2/neu in breast carcinoma is of prognostic significance, and is known to correlate with AIB1 overexpression. ${ }^{30,31}$ Although Her-2/neu is known to be increased in some endometrial carcinomas, its clinical prognostic value is not as clear. $^{30,31}$ Reports of Her-2/neu expression in endometrium are variable. Peiro et $a l^{32}$ found that Her$2 /$ neu amplification as measured by fluorescence in situ hybridization in endometrial carcinoma, correlated with non-endometrioid subtypes, higher grade and older age. In contrast, in our study, Her-2/neu protein detected by immunohistochemistry did not correlate with age, stage or histologic type. In addition, Her $/$ neu expression did not correlate significantly with AIB1 expression. Other studies have also found that, although Her-2/neu is overexpressed in up to $59 \%$ of endometrial carcinomas, it is not associated with prognosis or overall survival. ${ }^{30,31}$ Our results concur with these findings.

\section{Correlations between Biomarkers}

Previous studies have found a correlation between AIB1 and ER in endometrial carcinoma, and our findings support this correlation. ${ }^{23,25}$ However, we did not find a significant correlation between AIB1 and total PR or PR-B. It is interesting that AIB1 correlates with ER, but not with PR. This may indicate that AIB1 functions primarily with ER, but not PR. Indeed, it has now been shown that it is another member of this family, SRC-1, that modulates PR action in the mouse uterus. ${ }^{12,13}$ Estradiol is known to be a proliferative agent in the endometrium, whereas progesterone counteracts the proliferative effects of estradiol in the endometrial epithelium. The strongest association between the different markers in endometrial carcinoma in this study was between ER and PR-B. We demonstrate that ER and total PR are expressed more in carcinoma-associated normal endometrium than in carcinoma-associated complex atypical hyperplasia or endometrial carcinoma, and that these receptors are associated with lower stage and lower grade carcinomas, and younger age, in keeping with results from other studies. ${ }^{2,4}$

\section{Conclusions}

In summary, we show a significant correlation of high AIB1 expression with endometrial carcinoma using immunohistochemistry on tissue microarrays. We found a correlation between AIB1 and ER expression levels in endometrial carcinoma, but no significant correlation between AIB1 and PR. It is known that AIB1 is expressed at an increased level in breast carcinoma, ${ }^{33}$ and that the AIB1 protein interacts with ER to result in enhancement of estrogen-dependent transcription. ${ }^{14,33,34}$ Thus, an increase in AIB1 could cause increased sensitivity to estrogen exposure leading to endometrial hyperplasia and cancer. However, it is curious that we see even higher levels of AIB1 in Type II endometrial carcinoma than Type I endometrial carcinoma, since Type I carcinomas are more estrogen dependent. Perhaps, AIB1 is associated with an aggressive behavior of the cancer even if tumor cell proliferation is no longer driven by estrogen.

Future studies carried out to correlate AIB1 expression with endometrial carcinoma response to hormonal therapy and overall survival or disease recurrence would clarify the role that this nuclear receptor coactivator may play in guiding the treatment of endometrial carcinoma. It may be that use of inhibitors of the histone acetlytransferase activity associated with AIB1 mediated ER action could be useful in combination with progestin therapy or may benefit patients in whom progestin therapy is not effective.

\section{Acknowledgements}

We thank Norma Aumen for tissue array preparation, Nicole Manning for immunoblot analysis, 
Jason Hyde, MD and David Davis for IHC staining protocol guidance, Jan Lowery, $\mathrm{PhD}$ for statistical assistance and Lisa Litzenberger for photography.

This work was partially supported by a pilot grant from National Institutes of Health MD Anderson Gynecologic SPORE for Uterine Cancer Grant \# 5P50 CA098258 (MS/JR PIs).

Some of this work was presented as a poster at the United States and Canadian Academy of Pathology meeting in Atlanta, GA in February 2006.

\section{References}

1 Lax SF. Molecular genetic pathways in various types of endometrial carcinoma: from a phenotypical to a molecular-based classification. Virchows Arch 2004; 444:213-223.

2 Arnett-Mansfield RL, deFazio A, Wain GV, et al. Relative expression of progesterone receptors $A$ and $B$ in endometrioid cancers of the endometrium. Cancer Res 2001;61:4576-4582.

3 Ehrlich CE, Young PC, Stehman FB, et al. Steroid receptors and clinical outcome in patients with adenocarcinoma of the endometrium. Am J Obstet Gynecol 1998;158:796-807.

4 Kleine W, Maier T, Geyer $\mathrm{H}$, et al. Estrogen and progesterone receptors in endometrial cancer and their prognostic relevance. Gynecol Oncol 1998;38:59-65.

5 Kraus WL, Weis KE, Katzenellenbogen BS. Inhibitory cross-talk between steroid hormone receptors: differential targeting of estrogen receptor in the repression of its transcriptional activity by agonist- and antagonistoccupied progestin receptors. Mol Cell Biol 1995;15:1847-1857.

6 Sakamoto T, Eguchi H, Omoto Y, et al. Estrogen receptor-mediated effects of tamoxifen on human endometrial cancer cells. Mol Cell Endocrinol 2002;192:93-104.

7 O’Malley BW. A life-long search for the molecular pathways of steroid hormone action. Mol Endocrinol 2005;19:1402-1411.

8 Clark BF. The effects of oestrogen and progesterone on uterine cell division and epithelial morphology in spayed, adrenalectomized rats. J Endocrinol 1971;50: 527-528.

9 Tachi C, Tachi S, Lindner HR. Modification by progesterone of oestradiol-induced cell proliferation, RNA synthesis and oestradiol distribution in the rat uterus. J Reprod Fertil 1972;31:59-76.

10 Fernandez-Valdivia R, Mukherjee A, Mulac-Jericevic $\mathrm{B}$, et al. Revealing progesterone's role in uterine and mammary gland biology: insights from the mouse. Semin Reprod Med 2005;23:22-37.

11 Mulac-Jericevic B, Mullinax RA, DeMayo FJ, et al. Subgroup of reproductive functions of progesterone mediated by progesterone receptor-B isoform. Science 2000;289:1751-1754.

12 Han SJ, DeMayo FJ, Xu J, et al. Steroid receptor coactivator (SRC)-1 and SRC-3 differentially modulate tissue-specific activation functions of the progesterone receptor. Mol Endocrinol 2006;20:45-55.

13 Han SJ, Jeong J, Demayo FJ, et al. Dynamic cell type specificity of SRC-1 coactivator in modulating uterine progesterone receptor function in mice. Mol Cell Biol $2005 ; 25: 8150-8165$.
14 Labhart P, Karmakar S, Salicru EM, et al. Identification of target genes in breast cancer cells directly regulated by the SRC-3/AIB1 coactivator. Proc Natl Acad Sci USA 2005;102:1339-1344.

15 Giangrande PH, Kimbrel EA, Edwards DP, et al. The opposing transcriptional activities of the two isoforms of the human progesterone receptor are due to differential cofactor binding. Mol Cell Biol 2000; 20:3102-3115.

16 Sartorius CA, Shen T, Horwitz KB. Progesterone receptors $A$ and $B$ differentially affect the growth of estrogen-dependent human breast tumor xenografts. Breast Cancer Res Treat 2003;79:287-299.

17 Bouras T, Southey MC, Venter DJ. Overexpression of the steroid receptor AIB1 in breast cancer correlates with the absence of estrogen and progesterone receptors and positivity for p53 and HER2/neu. Cancer Res Feb 2001;61:903-907.

18 Oehler MK, Brand A, Wain GV. Molecular genetics and endometrial cancer. $\mathrm{J} \mathrm{Br}$ Menopause Soc Mar 2003;9:27-31.

19 Latta E, Chapman WB. PTEN mutations and evolving concepts in endometrial hyperplasia. Curr Opin Obstet Gynecol 2002;14:59-65.

20 Fleming FJ, Myers E, Kelly G, et al. Expression of SRC1, AIB1, and PEA3 in HER2 mediated endocrine resistant breast cancer; a predictive role for SRC-1. J Clin Pathol 2004;57:1069-1074.

21 Iwase $\mathrm{H}$, Omoto $\mathrm{Y}$, Toyama $\mathrm{T}$, et al. Clinical significance of AIB1 expression in human breast cancer. Breast Cancer Res Treat 2003;80:339-345.

22 Leslie KK, Stein MP, Kumar NS, et al. Progesterone receptor isoform identification and subcellular localization in endometrial cancer. Gynecol Oncol 2005; 96:32-41.

23 Gregory CW, Wilson EM, Apparao KB, et al. Steroid receptor coactivator expression throughout the menstrual cycle in normal and abnormal endometrium. J Clin Endocrinol Metab 2002;87:2960-2966.

24 Vienonen A, Miettinen S, Blauer M, et al. Expression of nuclear receptors and cofactors in human endometrium and myometrium. J Soc Gynecol Invest 2004;11: 104-112.

25 Uchikawa J, Shiozawa T, Shih HC, et al. Expression of steroid receptor coactivators and corepressors in human endometrial hyperplasia and carcinoma with relevance to steroid receptors and Ki-67 expression. Cancer 2003;98:2207-2213.

26 Kershah SM, Desouki MM, Koterba KL, et al. Expression of estrogen receptor coregulators in normal and malignant human endometrium. Gynecol Oncol 2004;92:304-313.

27 Glaeser M, Floetotto T, Hanstein B, et al. Gene amplification and expression of the steroid receptor coactivator SRC3 (AIB1) in sporadic breast and endometrial carcinomas. Horm Metab Res 2001;33: 121-126.

$28 \mathrm{Xu} \mathrm{J}, \mathrm{Li}$ Q. Review of the in vivo functions of the p160 steroid receptor coactivator family. Mol Endocrinol 2003;17:1681-1692.

$29 \mathrm{Xu}$ J, Liao L, Ning G, et al. The steroid receptor coactivator SRC-3 (p/CIP/RAC3/AIB1/ACTR/TRAM-1) is required for normal growth, puberty, female reproductive function, and mammary gland development. Proc Natl Acad Sci USA 2000;97:6379-6384.

30 Backe J, Gassel AM, Krebs S, et al. Immunohistochemically detected HER-2/neu-expression and 
prognosis in endometrial carcinoma. Arch Gynecol Obstet 1997;259:189-195.

31 Khalifa MA, Mannel RS, Haraway SD, et al. Expression of EGFR, HER-2/neu, P53, and PCNA in endometrioid, serous papillary, and clear cell endometrial adenocarcinomas. Gynecol Oncol 1994; 53:84-92.

32 Peiro G, Mayr D, Hillemanns P, et al. Analysis of HER$2 /$ neu amplification in endometrial carcinoma by chromogenic in situ hybridization. Correlation with fluorescence in situ hybridization, HER-2/neu, p53 and Ki-67 protein expression, and outcome. Mod Pathol 2004;17:227-287.

33 Anzick SL, Kononen J, Walker RL, et al. AIB1, a steroid receptor coactivator amplified in breast and ovarian cancer. Science 1997;277:965-968.

34 Anzick SL, Azorsa DO, Simons Jr SS, et al. Phenotypic alterations in breast cancer cells overexpressing the nuclear receptor co-activator AIB1. BMC Cancer 2003;3:22. 